Corresponding author: Paul Capdeville-Cazenave Dental Center for Innovation and Advanced Specialties, Calle de Albarracín, 35, 28037 Madrid, Spain Email: pcapdevicaz@gmail.com 0034628709408

Received: March 18, 2020

Accepted: October 06, 2020

\section{Attractive perception of profile class II patients treated with extractions versus dentoalveolar mandibular advancement devices}

Paul Capdeville-Cazenave ${ }^{1, *}$, Iván Nieto Sánchez ${ }^{1}$, Inés Díaz Renovales ${ }^{1}$, Javier de la Cruz Pérez ${ }^{1}$

Aim: This study aimed to compare the esthetic perception of profile silhouette of pictures of class II patients before and after treatment (extractions or mandibular advancement), according to a visual analog scale (VAS) among orthodontists, general dentists and lay people. Methods: A presentation of 18 class II adult patients silhouette was shown to three groups of participants (25 orthodontists, 25 general dentists, and 25 lay people) in two phases in an cross-sectional survey study. An initial presentation pre-treatment and a second one post-treatment, one month later. The presentation consisted of nine pictures of four extractions orthodontic cases (two maxillary premolars and two mandibular premolars): five males, four females, and other nine pictures with a dentoalveolar mandibular advance (Forsus ${ }^{\circledR}$ and/or class II elastics): four males, five females. To compare pre and post-treatment scores, an ANOVA test was performed. Independent variables studied were: sex, age, and previous or present orthodontic treatment of participants. Results: A total of 75 of evaluators scored 18 patient profiles before and after treatment. In the three groups, the post treatment silhouette scored significantly higher. Advancement treatment scored significantly higher than extractions, especially among lay people. Orthodontist gave the lowers score regardless of treatment. No differences were found between male and female scoring ( $p>0.05)$. The participants between $30-39$ years old gave higher scores than other age groups $(p<0,05)$. Conclusion: In our sample, class II orthodontic treatments did always improve esthetic perception of patients profile. Advancement treatment achieved a better esthetic perception than extractions, especially among lay people.

Keyword: Orthodontics. Malocclusion, Angle Class II. Esthetics. Cross-Sectional Studies. 


\section{Introduction}

The controversy remains in literature as to whether lay people or orthodontics, general dentist, and other dental professionals agree on their perception of facial attractiveness. According to Peretta et al. ${ }^{1}$, a symmetrical face is more attractive.

The skeletal Class II malocclusion is most often found in orthodontic practice after class I, according to Profitt ${ }^{2}$ and Simmons et al. ${ }^{3}$ with a prevalence of $15 \%$ in the population. In non-growing patients with skeletal class II discrepancies, the orthodontist is often faced the choice of either carrying out surgery or camouflage treatment. In this scenario, orthodontists have two dentoalveolar treatment choices, class II extractions or class II advancement devices (Forsus ${ }^{\circledR}$ device, class II elastics).

Modern society places a strong emphasis on physical attractiveness and facial beauty. The face remains one of the key features for determining human physical attractiveness ${ }^{4}$. One reason that motivates patients to seek orthodontic treatment is to improve facial aesthetics. Orthodontic treatment can influence facial aesthetics in a number of different ways, including well-aligned teeth ${ }^{5,6}$, an attractive smile $e^{6,7}$ and a pleasing facial profile $e^{7-9}$.

Subsequently, several studies have evaluated lip profile preferences ${ }^{8,9}$, where orthodontists and lay people have been requested to choose what they consider as most pleasant from groups of pictures or silhouettes profiles.

Peck and Peck ${ }^{9}$ in 1970, found that public preferred profiles with lips that were consistently fuller and more prominent, which are considered as ideal by orthodontists. According to Yehezkel and Turley ${ }^{10}$ cephalometrics standards show that the public now prefers a more complete and more convex facial profile. Foster et al. ${ }^{11}$ found that lay people chose profiles with fuller lips in children than in adults, what is consistent with the fact that lip profiles became more retruded with age, and were preferred over slightly fuller lips. Czarnecki et al. ${ }^{12}$ also reported that subjects preferred fuller women lips, but said that lips were closer and linked to the positions of the nose and chin.Subjects prefer a profile of protruded lips with a big nose and advanced chin. Lai et al. ${ }^{13}$ supported the importance of balancing the relations of the nose and lips. Abu Arqoub and Al-Khateeb ${ }^{14}$ stated that images with increased lower facial proportions were considered less attractive than corresponding images with reduced lower facial proportions, and those with Class II profile features were considered to be less attractive than corresponding images with Class III profile features.

Lips and chin are paramount to orthodontic patients to decide whether extract or not. These principles have become commonly accepted and used in contemporary orthodontic treatment planning.

The aim of this research was:

Firstly, to evaluate and score the differences, on a scale visual analog (VAS), of the perceived attractiveness among orthodontists, general dentists, and those outside field of the dental sciences, in class II patients, between the beginning and the end of treatment. 
Secondly, to compare how score changes a visual analog scale (VAS) of perceived attractiveness was used to compare between treatment with extractions and treatment with mandibular advancement among class II patients prior to the start and at the end of treatment time.

\section{Materials and methods}

The present study was approved by the Committee of Ethics in Research of Alfonso X University (Madrid, Spain) (protocol approval no. 2015-44/011), All participants signed an informed consent form.

Sample size was calculated based on a pilot study. A minimum of 25 subjects was required for each group of evaluators (orthodontists, dentists and laypeople) This cross sectional survey study consists of two populations: patients and reviewers.

Patients population: the study population consisted of photographs of patients who were treated with orthodontics and presented skeletal Class II malocclusion (according to cephalometric Steiner ANB).

Inclusion criteria were:

- Patients with skeletal class II.

- Patients treated either with extraction or advancement mandibular devices (Forsus ${ }^{\circledR}$ and or class II elastics in adults).

- Patients between 13 and 35 years old. As they were only silhouette tracings, the age of the patients could not be known by the participants.

- Evaluations of facial profiles were made from tracings of the soft tissue contour obtained from the lateral cephalograms from the $\mathrm{G}^{\prime}$ point to the Me point. Tracings were scanned in JPEG format, at a 1:1 ratio, with 300 dpi, using an Officejet 6500 scanner. The Frankfurt plane was oriented parallel to a true horizontal plane, and tracings were filled with black color using Photoshop (c) CS6 software (Adobe Systems).

Inclusion criteria were:

- Patients with skeletal class I or class III.

- Patients not treated either with extraction or advancement mandibular devices (Forsus ${ }^{\circledR}$ and or class II elastics in adults)

- Patients younger than 13 or older than 35 years old ).

- Participants were divided into three groups:

Group I: Orthodontists of Master of Orthodontics at Alfonso X el Sabio University (Madrid, Spain)

Group II: dentists of the Clinic of Dental Specialties at Alfonso X el Sabio University (Madrid, Spain).

Group III: Patients attending the Clinic of Dental Specialties at Alfonso X el Sabio University (Madrid, Spain). 
The dependent variable of the study was the aesthetic perception of the profile of patients with skeletal class II malocclusion assessed by a VAS scale of 0 to 10 (0 less attractive, 10 most attractive silhouette).

Independent variables

The studied independents variables were:

- Sex.

- Facial Biotype : according to Ricketts cephalometric study.

- The initial and final incisors inclination, given the involvement they have on the degree of protrusion or retraction of lips.

- Patient age in years.

The independent variables were considered for the group of evaluators were:

- Sex.

- $\quad$ Age of the evaluators.

- Orthodontic treatment of the participant (previous, at the time of the survey or non-orthodontic treatment at all) :

All the patients were treated with one of this two options:

- Patients treated with class II advancement device. Within this group patients treated with Forsus ${ }^{\circledR}$ were inlcuded.

- $\quad$ Class II skeletal patients treated with extractions of first upper and lower premolars (teeth 1.4, 2.4, 3.4, 4.4). And at the end of treatment which ended in molar and canine Class 1.

Patients' pictures were obtained from the medical records owned by the Master of Orthodontics clinic at Alfonso X el Sabio University (Madrid, Spain) since 2005. All patients had previously signed their consent for using their medical records for educational purposes and for publishing in dental journals or congresses.

Profile photos collected from the patients were taken with a Reflex device objective of $60 \mathrm{~mm}$ or more and ring flash described above. Those photos were taken with a manual mode camera photo with an ISO of 100 and F 10 or less inside a room with natural light without overexposure of light.

Once the photos were selected, they were cropped with Adobe Photoshop 6.0 version 13 software package (Adobe Systems, San Jose, Calif) Among all the photos, only the outer contour of the profile photo, from the base of the bridge of the nose to the cervical region, was selected. Photos were cut in a way that the profile end result was a black line representing these outer contours describing the face of the patient on a white background.

A questionnaire was given initially to the three groups of examiners to score profile photos of the 18 patients pretreatment that afterward were treated Class II. Nine before advancement devises and extractions (Figure 1 and Figure 2) and other nine 
before (Figure 3 and 4) and both, the questionnaire sheet to fill using a VAS visual scale and informed consent, were given to the participants before starting the study in the three groups.

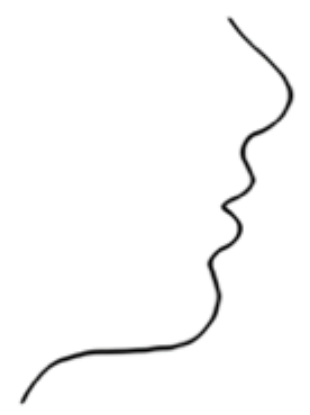

Figure 1. Silhouette of advancement devices pre treatment.

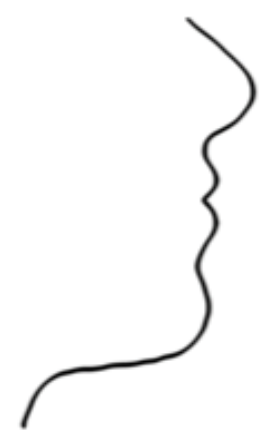

Figure 2. Silhouette of advancement devices post treatment.

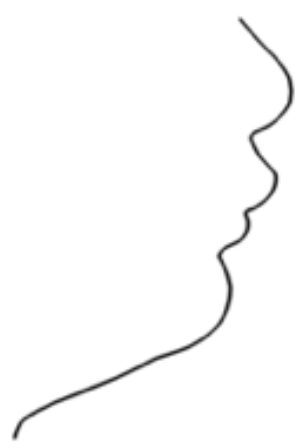

Figure 3. Silhouette of class II premolars extractions pre treatment. 


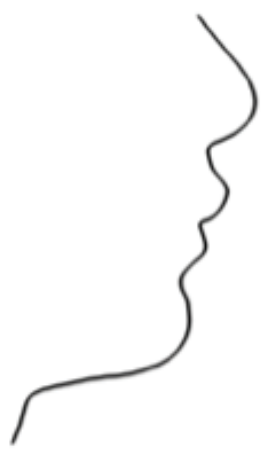

Figure 4. Silhouette of class II premolars extractions post treatment.

Participants were asked to evaluate the attractiveness of the presented silhouettes before treatment. A month later, the same participants were required to evaluate the presented silhouettes from the same patients after orthodontic treatment (extractions or dentaolveolar mandibular advancement).

Esthetics was evaluated through a Visual Analog Scale, where 0 was the lowest value (or the least aesthetic), and 10 was the highest (or the most aesthetic)

After collecting the pre-treatment profiles questionnaires, a second one was given to the same three groups one month later for post-treatment profiles evaluation.

Analysis was carried out using the SPSS software (v. 16.0, SPSS Inc., Chicago, IL, USA). Descriptive statistics was used to describe the distribution of data, and means and standard deviations were calculated.

The following comparisons were also made:

Comparison of scores before and after treatment were performed by t test of Student for related samples, such as descriptive statistics mean and standard deviation (SD) of the difference of pre-post treatment is presented.

These comparisons have been made for the total assessments and separately for pretreatment and post-treatment assessments.

Comparison of the score of both treatments (Advancement vs extractions) by t test of student's t-test for independent data. Comparisons were made in both, pretreatment and the post-treatment and in the difference between them, descriptive statistics as the mean (SD) are presented.

Comparison between categories of each of the variables that describe the evaluators were carried out with T-test for independent data if the variable is dichotomous (sex), or by analysis of variance ANOVA if the variable has tree or more categories (age, profession and background orthodontic).

The calculation of the sample size has been designed to detect differences in the aesthetic evaluation between groups of at least 1.5 points, considering a standard deviation of 1.9 points. 
The precision level $(1-\alpha)=95 \%$, and a statistical power $(1-\beta)=80 \%$. A sample of 25 evaluators will be required in each group.

This Comparisons are both pre and post-treatment and in the difference between them. Descriptive statistics such as the mean (SD) is presented and the adopted level of significance for this study was $p<0,01$.

\section{Results}

Differences between pre and post-treatment as well as differences between groups of evaluators (lay people, orthodontists and dentists) were evaluated. Significant differences were observed in the three cases (Figure 5) ie. Example: The mean (SD) score prior to the treatment was 4.8 (SD \pm 2.55$)$ in treatments with "Advancement" and $3.3(S D \pm 2,17)$ in treatments with "extractions", this difference was highly significant $(p<0.001)$.

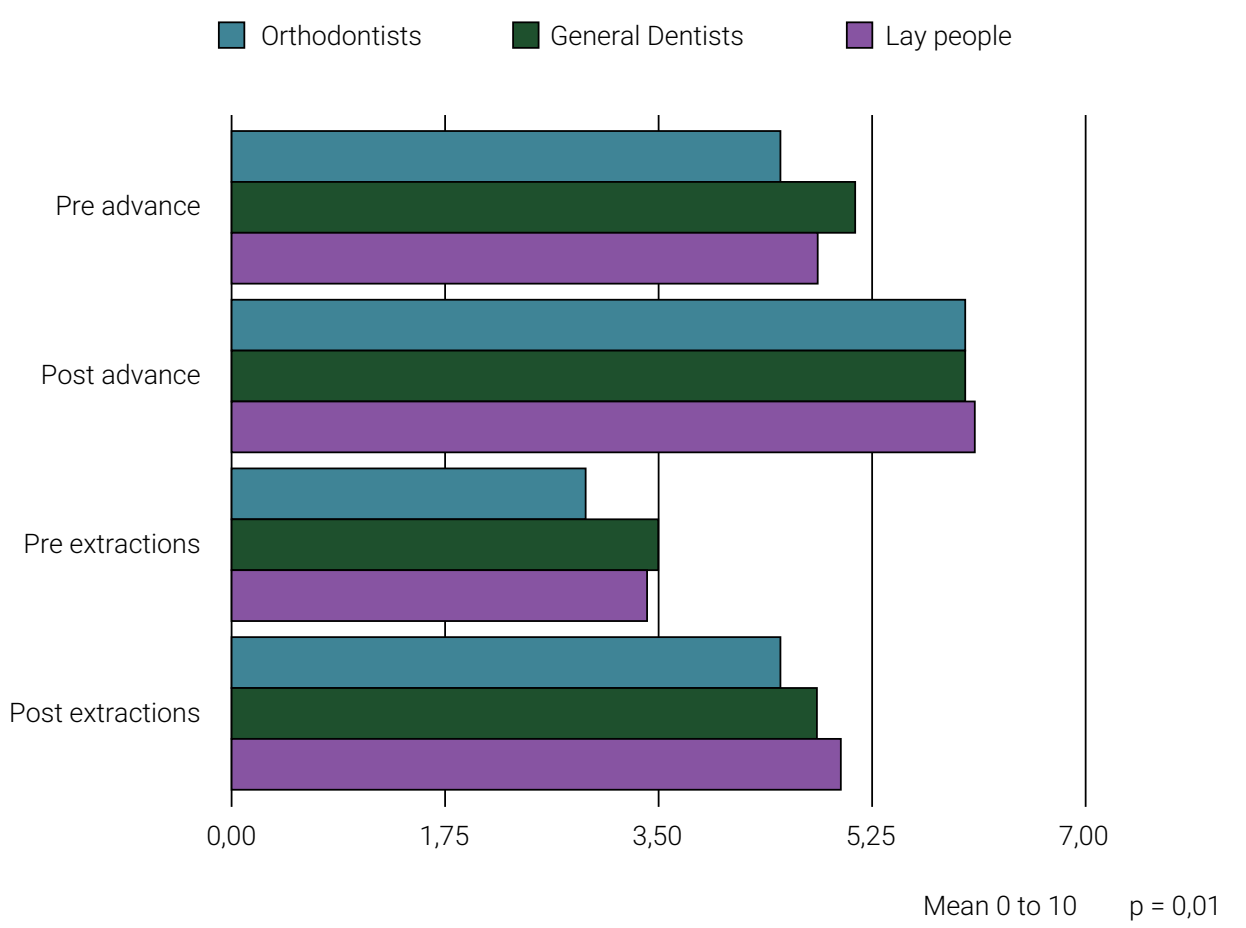

Figure 5. Esthetic scoring as per evaluator group.

\section{According to the sex of the evaluator.}

The differences after-before treatment are significantly higher in both, the total sample, and separately in both treatments, with the highest score post-treatment.

Advancement patient scores are considerably higher in advance treatment, even before any intervention. In the case of male, the difference pre-post in extraction treatment is significantly higher, while this difference has not been appreciated in the case of female patients. 
Only in pre-treatment of the total sample extraction and treatment of case, there are significant differences in scores associated with the sex of the evaluators, being male evaluators the ones who scored highest.

\section{Depending on the age of the evaluator.}

In all age groups, differences after-before are very significant in both, the total sample, and separately in both treatments, with the highest score after appropriate intervention.

In all age groups are scored higher in patients treated with advance, even before the intervention. Although there are some differences in scoring related to age, there is no clear trend.

\section{According to the evaluator profession.}

In all profession groups, differences after-before are $(<0.0001)$ in both, the total sample, and separately in both treatments, with the highest score after appropriate intervention.

There are significant differences in scoring related to professional groups among patients treated with advance. These one got higher scoring than extraction treatment, even before the intervention. Except in the case of "lay people" there is not relationship found with treatment in post-pre differences (Figure 6).

In most of the other comparisons, there are significant differences associated with the score given by profession group, being generally orthodontists. This group was the one giving the lowest scores.

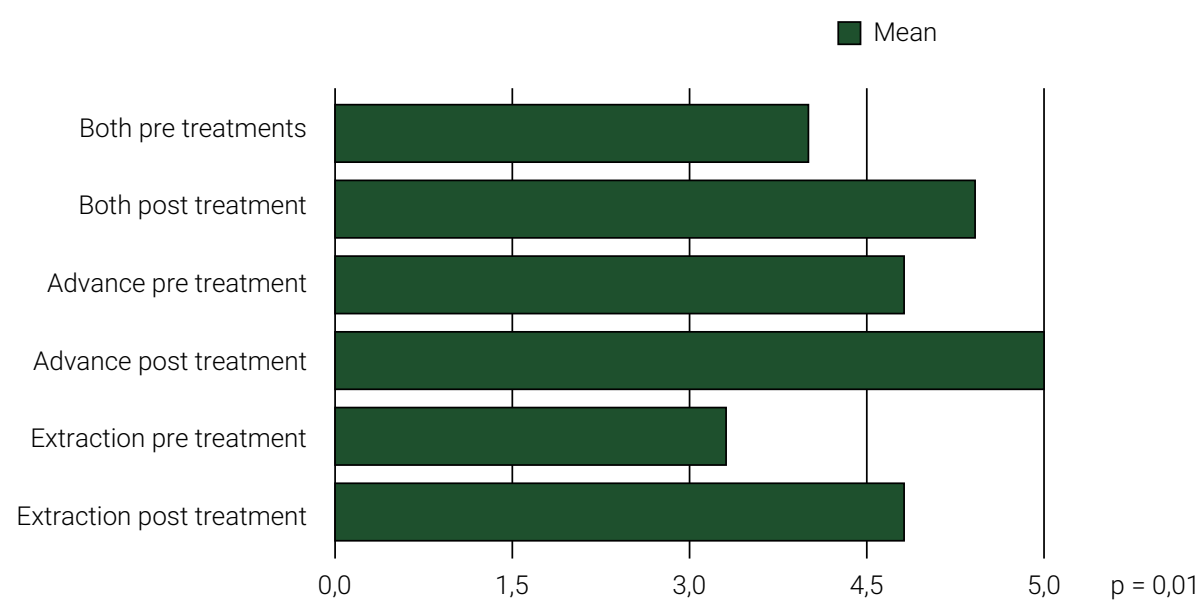

Figure 6. Esthetic scoring as per treatments.

\section{According to personal orthodontic experience of the evaluator.}

Regardless of the personal dental knowledge, differences between after-before are very significant in both, the total sample, and separately in both treatments, with the highest score after treatment. 
In all groups, patients treated with advance scored higher. Only in the case of those who never had orthodontic treatment there is not direct link with the post-pre differences, being greater the difference in the case of extractions.

But for post-treatment evaluations in all other comparisons no significant differences in scores associated with the group of personal orthodontic experience were found.

\section{Discussion}

Mergen et al. ${ }^{15}$ found, as in our study, that most attractive profiles occurred post-treatment but they did not find any remarkable differences depending on the type of treatment.

Also according to Nomura et al. ${ }^{16}$, an attractive soft tissue profile greatly varies among ethnicities and continents. In Europe, the preferred profile is usually flat or slightly protrusive. Therefore, extractions and advancement treatment could be both solutions that are socially accepted. Nevertheless, in our sample, although both treatments are considered esthetically accepted, advancement profiles were scored higher.

Peck and Peck' , A protruded lower lip was found more attractive in the case of female. However, the opposite happens for women's upper lip, that feature is considered unattractive, being attractive among men.

Cassia Molina de Paula et al. ${ }^{17}$ explain that the treatment of Class II patients performed with a combination of mandibular protraction appliance and fixed corrective orthodontics, had a positive effect on how orthodontists and laypeople were perceiving facial silhouettes, being this effect more frequently identified by laypeople.

Moresca et al. ${ }^{18}$ focused on Class II malocclusion treatment using the Herbst appliance, concluding that may produce a more esthetically improved facial profile silhouette compared with the one using the Forsus appliance. Our results did not compare skeletal but only dentoalveolar advancement devices, though.

For Rocha et al. ${ }^{19}$ the orthodontic treatment of Class II division 1

malocclusion both with extraction of maxillary first premolars and with the Forsus appliance had positive effects on facial profile esthetics. This was evaluated by both lay examiners and orthodontists, who assigned greater scores to the posttreatment profile.

Both treatment protocols produced similar results on the facial profile esthetics.

The group of orthodontists assigned higher scores to both pre- and posttreatment profiles than lay individuals, which is slightly different to our results.

In both studies ${ }^{18,19}$ results are similar to ours: Class II Treatment had positive effect in both cases.

In this same way, mandible advancement profiles produced more esthetic results than upper and lower extractions in our sample.

Conversely, it has been reported that the great benefit of treatment by extraction is achieved in cases with a greater dental protrusion, marked crowding, and thick and protruded lips. However, in individuals presenting a thin upper lip and an increased 
nasolabial angle at pretreatment, extraction may affect the upper lip positioning negatively and increase the nasolabial angle ${ }^{20}$.

Orthodontists used to measure changes in lateral cephalometrics and to evaluate the sagittal plane of patients is skeletal class, molar or canine class. However, in real life and in everyday social interactions, orthodontic result is rated from a face to face interaction rather than from a lateral view.

One of the improvements that should be developed in the future would be assessing changes of patients profile treated with extractions or with mandibular advancement through CBCT. It would also be interesting to evaluate the atractiveness of a 3D profile patient before and after treatment with different treatment modalities trough a stereophotogrammetry system.

It would also be a good option to compare the profiles treated with molar distalization in class II or the differences between female and male patients, in order to know what treatment achieves the most attractive profiles among the three groups of examiners. Another study line to develop in the future could be applying this type of study in class III, class I with open bite or excessive overbite patients.

This study has some limitations. This study included patients with 13-year-old or older. However, orthognathic surgery is usually performed after the patient's skeletal maturation period, which occurs in individuals aged 18 years. Thus, the perception of patients below this age by dental professionals may generates a bias assessment on the volume and position of the lip and chin. Also the extracted tooth may be an issued as differences in profile between first and second premolar extraction cases.

In our study, participants were asked to sit down and to fill in a paper form to carry out the study. This request may limit the motivation of certain evaluators that could have been reduced in case of using computerized questionnaire.

The fact that the study was only made up of 2D profile photos, could lead to think that a study with 3D photos could have been more suitable. Also the mood and state of mind of those evaluating at the time of giving scores could also have an effect on the results.

In conclusion, the difference scoring after-before treatment are significant, all pictures systematically scored higher after treatment.

Dentoalveolar advancement treatment is clearly preferred to extractions both in case of posttreatment. However, the advantage of the advance against extractions as to the difference in posterior and anterior to the intervention score, only becomes significant in all evaluations in of the male group, laypeople and those who they never underwent orthodontic treatment.

Orthodontists treating class II patients should consider not only the esthetic but also the inclination of lower incisor, crowding, and the ability to cooperate during treatment among other concerns and have a thorough chat with the patients explaining in full detail the advantages and disadvantages of extractions and dentoalveolar advancement. Having this said, both treatments offer an improvement in esthetics, that is slightly higher for non-extraction treatment. 


\section{References}

1. Perretta DI, Burt MD, Penton-Voak IS, Lee KJ, Rowland DA, Edwards R. Symmetry and Human Facial Attractiveness. Evol Hum Behav.1999; 20:295-307.

2. Proffit WR. Contemporary orthodontics. Madrid: Elsevier; 2005.

3. Simmons HC 3rd, Oxford DE, Hill MD. The prevalence of skeletal Class II patients found in a consecutive population presenting for TMD treatment compared to the national average. J Tenn Dent Assoc. 2008 Fall;88(4):16-8; quiz 18-9.

4. Riggio RE, Widaman, KF, Tucker JS, Salinas C. Beauty is more than skin deep: components of attractiveness. Basic Appl Soc Psych.1991;12(4):423-39. doi: 10.1207/s15324834basp1204_4.

5. Giddon DB. Orthodontic applications of psychological and perceptual studies of facial esthetics. Semin Orthod. 1995 Jun;1(2):82-93. doi: 10.1016/s1073-8746(95)80095-6.

6. Orsini MG, Huang GJ, Kiyak HA, Ramsay DS, Bollen AM, Anderson NK, Giddon DB. Methods to evaluate profile preferences for the anteroposterior position of the mandible. Am J Orthod Dentofacial Orthop. 2006 Sep;130(3):283-91. doi: 10.1016/j.ajodo.2005.01.026.

7. Sarver DM. The importance of incisor positioning in the esthetic smile: the smile arc. Am J Orthod Dentofacial Orthop. 2001 Aug;120(2):98-111. doi: 10.1067/mod.2001.114301.

8. Lines PA, Lines RR, Lines CA. Profilemetrics and facial esthetics. Am J Orthod 1978 Jun;73(6):648-57. doi: 10.1016/0002-9416(78)90225-7.

9. Peck H, Peck S. A concept of facial esthetics. Angle Orthod. 1970 Oct;40(4):284-318. doi: 10.1043/0003-3219(1970)040<0284:ACOFE>2.0.CO;2.

10. Yehezkel S, Turley PK. Changes in the African American female profile as depicted in fashion magazines during the 20th century. Am J Orthod Dentofacial Orthop. 2004 Apr;125(4):407-17. doi: 10.1016/j.ajodo.2003.05.007.

11. Foster LA, Thomson WM, Locker D. Assessing the responsiveness of the CPQ11-14 in New Zealand adolescents. Soc Sci Dent. 2010;1:48-53.

12. Czarnecki ST, Nanda RS, Currier GF. Perceptions of a balanced facial profile. Am J Orthod Dentofacial Orthop. 1993 Aug;104(2):180-7. doi: 10.1016/S0889-5406(05)81008-X.

13. Lai J, Ghosh J, Nanda RS. Effect of orthodontic therapy on the facial profile in long and short vertical facial patterns. Am J Orthod Dentofacial Orthop. 2000 Nov;118(5):505-13. doi: $10.1067 / \bmod 2000.110331$.

14. Abu Arqoub SH, Al-Khateeb SN. Perception of facial profile attractiveness of different anteroposterior and vertical proportions. Eur J Orthod. 2011 Feb;33(1):103-11. doi: 10.1093/ejo/cjq028.

15. Nomura M, Motegi E, Hatch JP, Gakunga PT, Ng'ang'a PM, Rugh JD, et al. Esthetic preferences of European American, Hispanic American, Japanese, and African judges for soft-tissue profiles. Am J Orthod Dentofacial Orthop. 2009 Apr;135(4 Suppl):S87-95. doi: 10.1016/j.ajodo.2008.02.019.

16. Mergen JL, Southard KA, Dawson DV, Fogle LL, Casko JS, Southard TE. Treatment outcomes of growing Class II Division 1 patients with varying degrees of anteroposterior and vertical dysplasias, Part 2. Profile silhouette evaluation. Am J Orthod Dentofacial Orthop. 2004 Apr;125(4):457-62. doi: 10.1016/j.ajodo.2003.06.004.

17. Molina de Paula EC, de Castro Ferreira Conti AC, Siqueira DF, Valarelli DP, de Almeida-Pedrin RR. Esthetic perceptions of facial silhouettes after treatment with a mandibular protraction appliance. Am J Orthod Dentofacial Orthop. 2017 Feb;151(2):311-6. doi: 10.1016/j.ajodo.2016.06.038.

18. Moresca AHK, de Moraes ND, Topolski F, Flores-Mir C, Moro A, Moresca RC, et al. Esthetic perception of facial profile changes in Class II patients treated with Herbst or Forsus appliances. Angle Orthod. 2020 Feb 24. doi: 10.2319/052719-362.1. 
19. Rocha AD, Casteluci CEVF, Ferreira FPC, Conti AC, Almeida MR, Almeida-Pedrin RR. Esthetic perception of facial profile changes after extraction and nonextraction Class II treatment. Braz Oral Res. 2020 Jan 31;34:e003. doi: 10.1590/1807-3107bor-2020.vol34.0003.

20. Tadic N, Woods MG. Incisal and soft tissue effects of maxillary premolar extraction in class II treatment. Angle Orthod. 2007 Sep;77(5):808-16. doi: 10.2319/081706-336. 